Rakusin ${ }^{1}$ ) nimmt mit Recht Stellung gegen alle diese "Verseifungsversuche".

Wasserlösliche Oele. Ueber die Anforderungen, die man an diese Oele stellen muss, spricht Buschmann ${ }^{3}$. Heydn ${ }^{3}$ patentiert wasserlösliche Absorptionsverbindungen vonTeer

1) Petr.Rev. 1911, XXV, p. 263.

7 Oel. und Fett-Ztg. 1911, pp. 173, 189, 207.

o D. A. C. 19328. in flüssiger oder fester Form; die Akt.-Ges. f. Asphaltierung ${ }^{1}$ ) patentiert ein wasserlösliches, haltbares, formaldehydhaltiges Desinfektionsmittel aus Oel usw.

Sulfoverbindungen. Eine schwefelhaltige Petroleum-Emulsion ist das sog. Thiopetrol ${ }^{8}$ ).

1) D. R. P. 240482.

(Fortsetzung folgt.)

J) Seifens.ZZtg. 1911, p. 1142.

\title{
Das Verhalten von Oelfarben- und Emailfarben-(Lackfarben-)Anstrichen unter dem Einfluss von Wasser.
}

\author{
L. E. Andés.
}

Anstriche jedweder Art, sobald sie auf Gegenständen ausgeführt sind, die sich dauernd im Freien befinden, sind neben anderen Einflüssen (Hitze durch direkt strahlende Sonne oder der Wärme überhaupt, Kälte, Staub und aufgewirbeltem Sand $u$. 2.) auch denjenigen des Wassers in Form von Tau, Regen und Schnee ausgesetzt. Man muss also mit diesen Einwirkungen rechnen, und es ist insbesondere der Regen, der bei längerer Einwirkung den Anstrichen Schaden bringen kann. Bei Schnee ist dies weniger der Fall, da dieser entweder in einer verhältnismässig kurzen Zeit zu Wasser wird, das abläuft oder trocknet oder unter dem Einflusse einer niederen Temperatur mehr oder weniger fest gefriert und nicht mehr nass ist oder Wasser absondert.

Streng unterschieden von dieser Art der Einwirkung von Wasser müssen die Einflüsse werden, die letzteres ausübt, wenn Anstriche bez. mit Anstrichen versehene Gegenstände dauernd (durch Wochen und Monate) den Wirkungen von Wasser ausgesetzt sind, derart, dass sich solche unter Wasser befinden und während dieser ganzen Zeit nicht durch Einwirkung von Luft wieder trocken werden können.

Anstriche, die nur zeitweilig der Einwirkung des Wassers ausgesetzt sind, die von Niederschlägen getroffen oder auch zeitweise mit Wasser überschwemmt, dann aber wieder einige Zeit der Luft ausgesetzt bleiben, leiden darunter lange nicht so, wie bei der dauernden Einwirkung des Wassers. Natürlich ist es auch nicht gleichgültig, aus welchen Stoffen die Anstrichfarben zusammengesetzt sind, aber im allgemeinen kann man doch sagen, dass die öligen Bindemittel der Anstrichfarben, ebenso wie die lackartigen (mit Ausnahme der spirituösen Harzlösungen, solange sie nicht völlig erhärtet sind) diesen
Einwirkungen gut widerstehen, die rein öligen indes besser als die lackartigen, bei welchen letzteren es sehr auf die Beschaffenheit und nicht in letzter Linie auf den Gehalt an trocknendem Oel ankommt. Nur Anstrichfarben, bezw. Lackfarben, die als Bindemittel einen Aussenlack enthalten, werden unter dem Einflusse des Wassers nicht weiss, verlieren also clen Glanz nicht und behalten ihr gutes Aussehen.

Oelfarbenanstriche, die den Einfüssen des Wassers durch Niederschläge ausgesetzt sind, werden durch diese niemals in der Weise angegriffen, dass die Haltbarkeit dabei in Frage käme; auch tagelange Regen vermögen nicht die trockene Oelfarbenschichte zu erweichen, sie schwammig zu machen oder gar Anlass zu werden, dass sich der Anstrich von der Unterlage (Eisen beispielsweise) loslöst, sich Blasen bilden, die mit Wasser erfüllt sind oder der Film sich abziehen lässt, wie es der Fall ist bei Oelfarbenanstrichen, die dauernd unter Wasser gehalten werden. Es darf dabei auch nicht übersehen werden, dass die Wasserschichte, die sich bei andauerndem Regen bildet, stets nur eine ganz minimale (Bruchteile von Millimetern) ist und dass sie unter der geringsten Einwirkung eines Luftzuges oder der Wärme wieder trocknet. Man darf deshalb auch den anhaltenden Niederschlag niemals der von allen Seiten einwirkenden Wassermasse gleichstellen, wie sie vorhanden ist, wenn sich Gegenstände unter Wasser befinden. Die Zeit, die bei Niederschlägen in Betracht kommt, ist eine viel zu kurze, um eine Erweichung, also Wasseraufnahme, im Gefolge zu haben und Versuche, über die noch einiges gesagt werden wird, haben ergeben, dass selbst erweichte Farbenhäute, sobald sie trocken geworden sind, wieder an der Unterlage adhärieren und ebenso fest erscheinen, wie sie vor der Erweichung gewesen sind, 
Emailfarben verhaiten sich in genau der gleichen Weise, und da dieselben, wenn richtig hergestellt, auch unter dauerndem Einflusse des Wassers sich nicht verändern, so sind sie unter allen Umständen als gegen nur zeitweise $\mathrm{An}$ griffe des Wassers völlig unempfindlich zu bezeichnen. Auch irgendwelche elektrische Einwirkungen sind (bei Eisen) ganz ausgeschlossen, da diese ja nur dann auftreten können, wenn auch das Metall mit in Aktion treten kann.

Wesentlich verschieden von dem Verhalten der Anstriche gegen zeitweilige Einwirkung von Wasser ist aber dasjenige unter Wasser. Bekannt ist, dass Harzlösungen der Einwirkung des Wassers auf die Dauer widerstehen, und es soll daher nicht weiter auf deren Beständigkeit eingegangen werden. Weniger bekannt aber dürfte es sein, dass Oelfarbenanstriche, wenn sie eine angemessene Zeit (etwa 3 Tage bei $90^{\circ}$ ) im Ofen getrocknet worden sind, ihre Empfindlichkeit gegen Wasser fast ganz eingebüsst haben und unverändert bleiben. Derartige Anstrichproben wurden (auf Eisen) wochenlang in Wasser aufbewahrt, ohne dass sich irgendwelche Veränderungen zeigten, wie sie bei luftgetrockneten Anstrichen schon nach wenigen Tagen auftreten. Bemerkenswert ist hierbei auch die Erfahrung, dass selbst durch Jahre an der Luft aufbewahrte Oelfarbenanstriche immer noch die Neigung haben, Wasser aufzunehmen, zu erweichen und keinen Vergleich mit den ofengetrockneten aushalten können.

Streicht man Glasplatten und Eisenplatten mit reinem Leinölfirnis, lässt solche einige Tage an der Luft trocknen und bringt sie dann unter Wasser, so erweichen beide fast gleichmässig, bilden Blasen und lösen sich mit geringer Nachhilfe ab. Da Glas mit Wasser wohl kaum einer elektrischen Einwirkung unterliegt, der Anstrich auf diesem aber sich genau so verhält, wie auf Eisen, so ist es vielleicht doch möglich, dass, ehe die Anstrichschichte vom Wasser durchdrungen und ein unmittelbarer Kontakt zwischen Metall und Wasser hergestellt ist, sich schon elektrische Einflüsse geltend machen können, die die weiteren Zerstörungen der Farbenhaut herbeiführen. Man kann natürlich die Ränder der Eisenplatten nicht derart durch den Anstrich schützen, dass von diesen aus nicht eine Berührung des Metalls mit Wasser möglich wäre, aber nachdem auch der Anstrich auf Glas erweicht, so sind die weiteren Erscheinungen bei Eisen wohl nur darauf zurückzuführen, dass das Metall durch den Oelfarbenanstrich hindurch mit dem Wasser in Kontakt getreten ist. Es steht durch ungezählte Versuche fest, dass selbst eine gut getrocknete (an der
Luft) Oelfarbenschichte, ganz unabhängig von dem darin befindlichen Farbekörper (mit Ausnahme von Bleimennige), nach einer grösseren oder geringeren Anzahl von Tagen dauernd der Einwirkung des Wassers ausgesetzt, zunächst durch Aufnahme geringerer Wassermengen ein weiches pelziges Aussehen erlangt, dann immer weicher wird, sich Blasen bilden (die Wasser in sich schliessen), die Dimensionen des aufstehenden Films sich vergrössern und dieser endlich entweder vollständig oder doch in grösseren Fetzen sich ablösen bez. abziehen lässt. Ueberlässt man einen solchen erweichten Anstrich sich selbst (derart aufstellen, dass das Wasser leicht und schnell abläuft) so verdampft das Wasser, der Film glättet sich und legt sich wieder an das Eisen an, so dass unter günstigen Umständen der unter Wasser gewesene Anstrich sich nur sehr wenig von jedem anderen unterscheidet.

Das Mass der Erscheinungen: Erweichen, Blasenbilden, Loslösen und Abziehbarkeit von der Unterlage ist naturgemäss bei den einzelnen Farbekörpern (es ist bei allen Versuchen stets der in gleicher Weise bereitete Leinölfirnis verwendet worden) durchaus nicht gleich, aber es war bisher nicht möglich, irgendeine Gruppe von Körperfarben aufzustellen, die sich durch diese oder jene Erscheinung charakterisieren liesse.

Bei Objekten, die, von verschiedenartiger Gestalt, aber immer Körper bildend, mit Anstrichen versehen und unter Wasser gehalten werden, bildet der Anstrich eine zusammenhängende Decke, die keinerlei Lücken aufweist; es ist also gänzlich ausgeschlossen, dass Wasser einwirken kann, ehe nicht die Anstrichschichte Wasser aufgenommen und in weiterer Folge dieses bis auf das Metall gedrungen ist; ich halte es dernnach auch für unmöglich, dass die Beschädigungen der Anstrichschichten eine unmittelbare Folge elektrischer Ströme sein können - diese sind nur die mittelbare Folge der Durchdringung der Oelfarbenschichte mit Wasser.

Anstrichmassen, welche Harzlösungen als Bindemittel enthalten, werden von Wasser auch bei langandauernder Einwirkung in gar keiner Weise angegriffen und selbst Spirituslacke zeigen eine nicht geringere Beständigkeit, obwohl sie unter den Einflüssen der Atmosphärilien in verhältnismässig kurzer Zeit nicht allein den Glanz verlieren und rissig werden, sondern auch Neigung zum Loslösen zeigen. Es ist somit, was übrigens durch die Praxis längst erwiesen ist, jede Harzlösung in geringerem oder höherem Mass befähigt, Metall unter Wasser dauernd vor Korrosion zu schützen und elektrischen Einwirkungen entgegenzuwirken. 